ARTIGO ORIGINAL

\title{
Comparação de análises de amostras de falantes por meio de diferentes protocolos perceptivos-auditivo
}

\author{
Comparison of analysis of voice samples by different perceptive-hearing protocols
}

\author{
Luan Paulo Franco Magalhães ${ }^{1}$, Cresio de Aragão Dantas Alves ${ }^{2 *}$, Renata Christina Vieira ${ }^{3}$
}

\begin{abstract}
${ }^{1}$ Mestrando em Processos Interativos dos Órgãos e Sistemas, pela Universidade Federal da Bahia (UFBA), Graduado em Fonoaudiologia, pela Universidade do Estado da Bahia (UNEB). ; '2 Doutor em Medicina, pela UFBA. Professor Associado de Pediatria, Chefe do Serviço de Endocrinologia Pediátrica no Hospital Universitário Prof. Edgar Santos (FAMED/UFBA). ${ }^{3}$ Doutora em Linguística Aplicada e Estudos da Linguagem, pela Pontifícia Universidade Católica de São Paulo (PUC/SP). Mestre em Linguística, pela Universidade do Estado do Rio de Janeiro (UERJ).
\end{abstract}

\begin{abstract}
Resumo
A escala RASATI foi adotada, no Brasil, em 2002, e sua sigla corresponde, do ponto de vista anatomofisiológico e perceptivo-auditivo, a rouquidão (grau de): irregularidade; aspereza; soprosidade; astenia; tensão e instabilidade ${ }^{6}$. 0 protocolo VPAS (Vocal Profile Analysis Scheme) avalia a variedade de ajustes da qualidade vocal nos planos fonatório, articulatório e de tensão, assim como os elementos de dinâmica vocal, como pitch, loudness, taxa de elocução, pausas e suporte respiratório, de acordo com a fonética. Objetivo: comparar os resultados das análises perceptivo-auditiva dos protocolos da RASATI e do VPAS-PB. Metodologia: estudo qualitativo, com amostra de conveniência. As amostras de vozes dos 10 falantes do sexo masculino, com 34 a 39 anos, foram coletadas no Corpo de Bombeiros do estado do Rio de Janeiro. As amostras foram entregues para três juízes de VPAS-PB para a descrição do perfil individual. Em seguida, as amostras foram utilizadas para nova análise vocal, por meio do protocolo da RASATI, baseada na amostra do VPAS-PB, para assim possibilitar a comparação dos dados dos dois protocolos. Resultados: foi possível observar a maior descrição do perfil vocal com mais aspectos contemplados na utilização do protocolo do VPAS-PB, enquanto na RASATI observam-se características que se restringem aos aspectos fonatórios. Conclusão: o VPAS-PB permite ao profissional maiores chances de identificar o problema vocal dentro dos aspectos apresentados, por abordar tanto o trato vocal como a fonte glótica, que é a base do protocolo da RASATI. Esta última, por sua vez, permite que o avaliador identifique os aspectos fonatórios na prática clínica com maior velocidade, visto que aborda somente os aspectos que se referem à fonte glótica, mas não contempla os outros aspectos, como dinâmica vocal e trato vocal.
\end{abstract}

Palavras-chave: Voz. Protocolo. Avaliação Vocal. Fonética.

\begin{abstract}
The RASATI scale was adopted in Brazil in 2002, and its acronym corresponds, from the anatomophysiological and perceptualauditory point of view, to hoarseness (degree of): irregularity; roughness; breathiness; asthenia; tension and instability ${ }^{6}$. The Vocal Profile Analysis Scheme (VPAS) protocol evaluates the variety of vocal quality adjustments in the phonatory, articulatory, and tension planes, as well as the vocal dynamics elements such as pitch, loudness, speech rate, pauses, and respiratory support according to its phonetic perspective. Objective: to compare the results of the auditory-perceptual analysis of the RASATI and VPAS-PB protocols. Methodology: a qualitative study with a convenience sample. The voice samples of the 10 male speakers, aged 34 to 39 years old, were collected in the Fire Department at the state of Rio de Janeiro - Brazil and thus given to three VPAS-PB judges for individual profile description. Then, the samples were used for further vocal analysis, using the RASATI protocol, based on the VPAS-PB sample, in order to allow comparison of data from both protocols. Results: it was possible to observe the greater description of the vocal profile with more aspects contemplated in the use of the VPAS-PB protocol, while in RASATI there were characteristics that were restricted to the phonatory aspects. Conclusion: the VPAS-PB allows the professional greater chances of identifying the vocal problem within the presented aspects, by addressing both the vocal tract and the glottic source, which is the basis of the RASATI protocol. The latter, however, allows the evaluator to identify phonatory aspects in clinical practice with greater speed, since it only addresses aspects that refer to the glottic source, but does not address other aspects, such as vocal dynamics and vocal tract.
\end{abstract}

Keywords: Voice. Protocol. Vocal Evaluation. Phonetics.

\section{INTRODUÇÃO}

A partir da adaptação na atividade do aparelho digestório, assim como o do respiratório, ocorre a produção

Correspondente/Corresponding: *Cresio de Aragão Dantas Alves Instituto de Ciências da Saúde/UFBA. - End: Av. Reitor Miguel Calmon, s/n, Vale do Canela. 40110-100. Salvador, Ba - Tel: (71) 99178-4055 —E-mail: cresio.alves@uol.com.br dos sons da fala. O aparelho respiratório, por meio do controle do fluxo aéreo durante a expiração, participa do processo da produção da fala, pois gera uma corrente de ar egressiva, que é a base da geração das fontes de ruído e sonoridade. No que diz respeito aos sons das vogais, ele faz com que a laringe produza sonoridade, também chamado de vozeamento, devido às vibrações das pregas vocais. 0 aparelho digestório, por sua vez, contribui na qualidade 
de articuladores ativos, como lábios, língua, mandíbula e palato mole, além dos passivos, como dentes, palato duro e parede posterior da faringe. Além disso, contribui com os ressoadores, como a cavidade oral, nasal, faríngea e labial, que irão modificar o som produzido a nível glótico, possibilitando diversos segmentos vocálicos e consonantais ${ }^{1}$.

A produção da fala é baseada na teoria linear fonte-filtro, na qual a fonte é a vibração laríngea, e o filtro (sistema de transmissão seletiva ou sistema ressonador) é o trato vocal ${ }^{2}$. A laringe, por meio dos ciclos de abertura e fechamento das pregas vocais, é um transdutor de energia aerodinâmica (fluxo de ar) em acústica ${ }^{3}$.

$\mathrm{Na}$ fonte (sinal laríngeo), a análise restringe-se ao som produzido em nível de fonte glótica, relacionando-se a harmônicos e, também, à frequência fundamental. Esta análise considera as alterações da vibração da mucosa das pregas vocais. No filtro (sinal de saída), obtém-se uma análise mais ampla, a qual inclui também os aspectos vocais relacionados à ressonância e articulação. Esta avaliação vocal perceptiva é realizada ao considerar a passagem dos harmônicos produzidos pela fonte glótica através trato vocal (efeito de filtragem do som) ${ }^{4}$.

A avaliação perceptiva da voz é um procedimento muito utilizado na avaliação diagnóstica de distúrbios da voz, sendo padrão ouro na clínica vocal, porém a eficácia de seus resultados depende principalmente da experiência do avaliador. É, portanto, uma das melhores ferramentas de avaliação vocal, mas há possíveis julgamentos diferenciados devido à subjetividade dos avaliadores, por não padronizarem a terminologia usada, por exemplo, ou por fazerem uso de diferentes métodos de avaliação ${ }^{5}$.

A escala RASATI foi adotada em 2002, no Brasil, e sua sigla corresponde, do ponto de vista anatomofisiológico e perceptivo-auditivo, a rouquidão (grau de): irregularidade (aperiodicidade); aspereza (rigidez da mucosa que também causa alguma irregularidade vibratória); soprosidade (presença de ruído de fundo, audível e visível em diferentes regiões do espectro6); astenia (relacionada com o mecanismo de hipofunção das pregas vocais e reduzida energia de emissão do som); tensão (associada a esforço vocal por aumento da adução glótica); e instabilidade, sendo este último parâmetro (I - Instabilidade) acrescentado, em 2008, à escala previamente explicada ${ }^{7}$, traduzindo a flutuação das qualidades da voz avaliada ${ }^{4}$.

Sendo a avaliação perceptivo-auditiva da voz uma tarefa complexa, há diversos fatores que a influencia como a experiência do avaliador e suas preferências, tipo de estímulo (emissão sustentada, fala encadeada), treinamento que este avaliador possui ${ }^{89}$.

O protocolo VPAS (Vocal Profile Analysis Scheme), sua adaptação, tradução e validação para o Brasil, VPAS$-\mathrm{PB}$, avalia a variedade de ajustes da qualidade vocal nos planos fonatório, articulatório e de tensão, assim como os elementos de dinâmica vocal, como pitch, loudness, taxa de elocução, pausas e suporte respiratório, de acordo com a fonética. Este protocolo é resultante do modelo teórico proposto por Laver (1980), fruto do trabalho contínuo de equipe de pesquisadores no Queen Margareth University $(Q M U \text { - Edinburgh })^{10}$. A aplicação deste roteiro tem como resultado o perfil de qualidade vocal da amostra coletada ${ }^{5}$.

$O$ roteiro VPAS-PB analisa o setting, ou ajuste do indivíduo, e foi desenvolvido por meio do modelo fonético de qualidade vocal. $\mathrm{O}$ ajuste refere-se à postura fonatória ou articulatória mantida pelo falante por um período de tempo. Este roteiro permite uma avaliação perceptiva de qualidade vocal em termos das categorias de ajustes: de trato vocal, fonatórios, de tensão muscular e prosódicos. Sendo assim, há diversos graus que o VPAS-PB possibilita avaliar, variando de 1 a $6^{11}$.

OVPAS é o instrumento mais completo, que possibilita descrever diversos ajustes de trato vocal, por exemplo, em um indivíduo, além de fonatórios e de dinâmica vocal, tornando o perfil vocal do paciente mais detalhado e preciso. Ele permite caracterizar e descrever a qualidade vocal por meio de um conjunto de ajustes combinados e suas características. Estes ajustes, quando em diferentes combinações, modificam a qualidade de voz do falante ${ }^{11}$. Assim, o VPAS-PB é um instrumento complementar, que pode ser utilizado na identificação de falantes, traçando, portanto, seu perfil vocal com ampla descrição da qualidade da voz desses indivíduos identificados nas amostras de fala ${ }^{11}$.

Sendo assim, o objetivo dessa pesquisa é realizar uma comparação das 10 amostras de fala que foram previamente analisadas por meio do protocolo VPAS-PB (ANEXO 1), realizando nova análise dessas mesmas amostras por meio da RASATI (ANEXO 2), a fim de observar e refletir sobre o uso dos dois protocolos separadamente e compará-los.

\section{METODOLOGIA}

As amostras de vozes dos falantes foram coletadas no Corpo de Bombeiros do estado do Rio de Janeiro, após os participantes terem assinado o Termo de Consentimento Livre e Esclarecido (TCLE). O projeto de pesquisa foi submetido e aprovado no Comitê de Ética e Pesquisa, da Pontifícia Universidade Católica de São Paulo - PUC/SP, sob o número 2.153.565. Foram incluídos pacientes do sexo masculino, entre 34 e 39 anos, profissionais do Corpo de Bombeiros do Estado do Rio de Janeiro, que participaram da pesquisa cujo título é Identificação de falante: um estudo perceptivo da qualidade de voz. Foram excluídos pacientes com questões vocais ou auditivas; ou que fizessem uso de voz profissional; que fossem do sexo feminino; e que fossem pertencentes a outros estados.

As amostras foram entregues para três juízes, com experiência comprovada do protocolo VPAS-PB, para descrição do perfil individual. Após esse momento, os resultados foram utilizados para nova análise vocal, através da RASATI, para, dessa forma, possibilitar a comparação dos dados dos dois protocolos.

Trata-se de um estudo qualitativo, com amostras de conveniência, composto por dez sujeitos, militares do Corpo de Bombeiros, do sexo masculino, com idade entre $34 \mathrm{e}$ 39 anos, ensino médio completo, residentes do estado do 
Rio de Janeiro. A escolha do sexo masculino se deu por conta da prevalência desse gênero na autoria de delitos, visto que a pesquisa inicial feita com essas amostras se baseou em um estudo de perícia vocal e ao fato de que, devido às diferenças em termos de características acústicas da voz da mulher e do homem, seria necessária a formação de dois grupos distintos; o nível de escolaridade, a profissão e a naturalidade foram determinados para dar homogeneidade sociolinguística às amostras.

Os protocolos de coleta utilizados nesta pesquisa foram por meio de questionário e o protocolo VPAS-PB (ANEXO 1).

Os equipamentos utilizados para as gravações foram: gravador portátil Zoom H5, celular Samsung Galaxy A5, com sistema Android, processador Quad Core de $1,2 \mathrm{GHz}$, e o aplicativo de gravação telefônica Automatic Call Recorder. Cada amostra de fala foi gravada simultaneamente de duas maneiras: através do gravador e pelo aparelho celular através do aplicativo de gravação. As gravações foram realizadas na frequência de amostragem 44.100 Hz, 16 bits e em extensão wav, de acordo com as referências de estudos realizados no Laboratório Integrado de Análise Acústica e
Cognição (LIAAC), da PUC/SP.

Atendendo à Resolução № 466, de 12 de dezembro de 2012, este projeto segue os aspectos éticos, visando assegurar os direitos e deveres que dizem respeito aos participantes da pesquisa. Todos os achados de pesquisa, fatos ou informações encontradas no decorrer da mesma e que sejam considerados de relevância para os participantes ou comunidades participantes foram registrados.

\section{RESULTADOS}

O protocolo VPAS-PB apresenta diferentes aspectos a serem analisados, sendo eles os de trato vocal, fonatórios e dinâmica vocal. A RASATI, por sua vez, apresenta apenas aspectos fonatórios. A Tabela abaixo apresenta os resultados previamente analisados por meio do VPAS-PB, das amostras de indivíduos que participaram da pesquisa Identificação de falante: um estudo perceptivo da qualidade de voz, caracterizando todos os aspectos que este protocolo aborda. Além disso, a tabela apresenta a nova análise das mesmas amostras de falantes, porém esta última por meio da RASATI e os aspectos que este protocolo apresenta.

Tabela 1 - Comparação entre VPAS (ANEXO 1) e RASATI (ANEXO 2)

\begin{tabular}{|c|c|c|c|}
\hline $\begin{array}{c}\text { FALANTES } \\
\text { AUDIOGRAVADOS }\end{array}$ & ASPECTOS DO TRATO VOCAL & ASPECTOS FONATÓRIOS & ASPECTOS DA DINÂMICA VOCAL \\
\hline 1 (VPAS) & $\begin{array}{l}\text { Lábios com extensão diminuída grau } 2 \text {, mandíbula } \\
\text { aberta e com extensão diminuída em grau 1, corpo } \\
\text { de língua abaixado em grau 1, altura de laringe } \\
\text { abaixada em grau } 1 .\end{array}$ & $\begin{array}{l}\text { Voz crepitante em grau } \\
1 .\end{array}$ & $\begin{array}{l}\text { Pitch habitual abaixado em grau } 2 \text {, pitch } \\
\text { com extensão diminuída em grau } 2 \text {. }\end{array}$ \\
\hline \multirow[t]{2}{*}{1 (RASATI) } & & $\begin{array}{l}\text { Rouquidão: } 0 \\
\text { Aspereza: } 0 \\
\text { Soprosidade: } 0 \\
\text { Astenia: } 0 \\
\text { Tensão: } 0 \\
\text { Instabilidade: } 0\end{array}$ & \\
\hline & ASPECTOS DO TRATO VOCAL & ASPECTOS FONATÓRIOS & ASPECTOS DA DINÂMICA VOCAL \\
\hline 2 (VPAS) & $\begin{array}{l}\text { Lábios com extensão diminuída grau } 1 \text {, mandíbula } \\
\text { aberta em grau } 1 \text {, ponta de língua avançada em grau } \\
2 \text {, corpo de língua elevado em grau } 1 \text {, constrição } \\
\text { faríngea em grau 1, hiperfunção de trato vocal em } \\
\text { grau } 1 .\end{array}$ & & $\begin{array}{l}\text { Tremor e irregularidade laríngea e } \\
\text { continuidade interrompida. }\end{array}$ \\
\hline \multirow[t]{2}{*}{2 (RASATI) } & & $\begin{array}{l}\text { Rouquidão: } 0 \\
\text { Aspereza: } 0 \\
\text { Soprosidade: } 0 \\
\text { Astenia: } 0 \\
\text { Tensão: } 1 \\
\text { Instabilidade: } 1 \\
\end{array}$ & \\
\hline & ASPECTOS DO TRATO VOCAL & ASPECTOS FONATÓRIOS & ASPECTOS DA DINÂMICA VOCAL \\
\hline 3 (VPAS) & $\begin{array}{l}\text { Mandíbula aberta em grau } 1 \text {, ponta de língua } \\
\text { avançada em grau 1, corpo de língua abaixado em } \\
\text { grau } 1 \text {, altura de laringe abaixada em grau } 1 .\end{array}$ & $\begin{array}{l}\text { Voz crepitante em grau } 1 \\
\text { e áspera em grau2. }\end{array}$ & $\begin{array}{l}\text { Taxa de elocução rápida em grau } 2 \text { e } \\
\text { loudness habitual aumentado em grau } 1 .\end{array}$ \\
\hline 3 (RASATI) & & $\begin{array}{l}\text { Rouquidão: } 0 \\
\text { Aspereza: } 2 \\
\text { Soprosidade: } 0 \\
\text { Astenia: } 0 \\
\text { Tensão: } 0 \\
\text { Instabilidade: } 0\end{array}$ & \\
\hline
\end{tabular}


Luan Paulo Franco Magalhães, Cresio de Aragão Dantas Alves, Renata Christina Vieira

\begin{tabular}{|c|c|c|c|}
\hline $\begin{array}{c}\text { FALANTES } \\
\text { AUDIOGRAVADOS }\end{array}$ & ASPECTOS DO TRATO VOCAL & ASPECTOS FONATÓRIOS & ASPECTOS DA DINÂMICA VOCAL \\
\hline & ASPECTOS DO TRATO VOCAL & ASPECTOS FONATÓRIOS & ASPECTOS DA DINÂMICA VOCAL \\
\hline 4 (VPAS) & $\begin{array}{l}\text { Língua recuada em grau 1, corpo de língua recuado } \\
\text { em grau } 2 \text { e elevado em grau } 1 \text {, mandíbula com } \\
\text { extensão aumentada em grau 1, constrição faríngea } \\
\text { em grau 2, hiperfunção de trato vocal em grau } 2, \\
\text { hiperfunção laríngea em grau } 2 .\end{array}$ & $\begin{array}{l}\text { Voz crepitante em grau } 2 \\
\text { e áspera em grau } 2 .\end{array}$ & $\begin{array}{l}\text { Taxa de elocução lenta em grau } 1 \text { epitch } \\
\text { habitual abaixado em grau } 1 .\end{array}$ \\
\hline \multirow[t]{2}{*}{4 (RASATI) } & & $\begin{array}{l}\text { Rouquidão: } 0 \\
\text { Aspereza: } 2 \\
\text { Soprosidade: } \\
\text { Astenia: } 0 \\
\text { Tensão: } 2 \\
\text { Instabilidade: } 0\end{array}$ & \\
\hline & ASPECTOS DO TRATO VOCAL & ASPECTOS FONATÓRIOS & ASPECTOS DA DINÂMICA VOCAL \\
\hline 5 (VPAS) & $\begin{array}{l}\text { Lábios com extensão diminuída em grau 1, } \\
\text { mandíbula aberta em grau1, ponta de língua } \\
\text { avançada em grau1, corpo de língua com extensão } \\
\text { diminuída em grau } 1 \text {, constrição faríngea em grau1, } \\
\text { hiperfunção de trato vocal em grau } 1 .\end{array}$ & Voz áspera em grau 1. & $\begin{array}{l}\text { Taxa de elocução lenta em grau 1, pitch } \\
\text { com variabilidade diminuída em grau } 1 \text { e } \\
\text { pitch habitual elevado em grau 1. Loudness } \\
\text { habitual abaixado em grau } 1 \text { na leitura } \\
\text { de frase. }\end{array}$ \\
\hline \multirow[t]{2}{*}{5 (RASATI) } & & $\begin{array}{l}\text { Rouquidão: } 0 \\
\text { Aspereza: } 1 \\
\text { Soprosidade: } 0 \\
\text { Astenia: } 0 \\
\text { Tensão: } 1 \\
\text { Instabilidade: } 0 \\
\end{array}$ & \\
\hline & ASPECTOS DO TRATO VOCAL & ASPECTOS FONATÓRIOS & ASPECTOS DA DINÂMICA VOCAL \\
\hline 6 (VPAS) & $\begin{array}{l}\text { Lábios com extensão diminuída em grau } 1 \text { e } \\
\text { protraídos em grau 2, mandíbula com extensão } \\
\text { aumentada em grau 1, ponta de língua recuada em } \\
\text { grau1, corpo de língua abaixado em grau } 1 \text { e recuado } \\
\text { em grau 1, altura de laringe abaixada em grau } 1, \\
\text { hiperfunção de trato vocal em grau 1, hiperfunção } \\
\text { laríngea em grau } 1 .\end{array}$ & $\begin{array}{l}\text { Voz crepitante em grau } 1 \\
\text { e áspera em grau } 1 .\end{array}$ & $\begin{array}{l}\text { Pitch com extensão diminuída em grau } 1 \mathrm{e} \\
\text { com variabilidade diminuída em grau } 1 \text {. }\end{array}$ \\
\hline \multirow[t]{2}{*}{6 (RASATI) } & & $\begin{array}{l}\text { Rouquidão: } 0 \\
\text { Aspereza: } 1 \\
\text { Soprosidade: } 0 \\
\text { Astenia: } 0 \\
\text { Tensão: } 1 \\
\text { Instabilidade: } 0 \\
\end{array}$ & \\
\hline & ASPECTOS DO TRATO VOCAL & ASPECTOS FONATÓRIOS & ASPECTOS DA DINÂMICA VOCAL \\
\hline 7 (VPAS) & $\begin{array}{l}\text { Lábios estirados em grau 1, mandíbula com extensão } \\
\text { aumentada em grau 1, ponta de língua avançada } \\
\text { em grau } 2 \text {, corpo de língua avançado em grau } 1 \text { e } \\
\text { abaixado em grau 1, expansão faríngea em grau } 2, \\
\text { laringe abaixada em grau } 1 .\end{array}$ & & $\begin{array}{l}\text { Pitch com variabilidade aumentada em grau } \\
\text { 1, loudness habitual diminuído em grau } 2 .\end{array}$ \\
\hline \multirow[t]{2}{*}{7 (RASATI) } & & $\begin{array}{l}\text { Rouquidão: } 0 \\
\text { Aspereza: } 0 \\
\text { Soprosidade: } \\
\text { Astenia:0 } \\
\text { Tensão: } 0 \\
\text { Instabilidade: } 0\end{array}$ & \\
\hline & ASPECTOS DO TRATO VOCAL & ASPECTOS FONATÓRIOS & ASPECTOS DA DINÂMICA VOCAL \\
\hline 8 (VPAS) & $\begin{array}{l}\text { Lábios com extensão aumentada em grau 1, } \\
\text { mandíbula com extensão aumentada em grau } 1, \\
\text { ponta de língua avançada em grau 2, constrição } \\
\text { faríngea em grau } 1 \text {, altura de laringe elevada em } \\
\text { grau 2, hiperfunção laríngea em grau } 1 .\end{array}$ & $\begin{array}{l}\text { Voz soprosa em grau } 2 \text { e } \\
\text { áspera em grau } 1 .\end{array}$ & $\begin{array}{l}\text { Taxa de elocução rápida em grau } 1 \text { e pitch } \\
\text { habitual elevado em grau } 2 \text {. }\end{array}$ \\
\hline
\end{tabular}




\begin{tabular}{|c|c|c|c|}
\hline $\begin{array}{c}\text { FALANTES } \\
\text { AUDIOGRAVADOS } \\
\end{array}$ & ASPECTOS DO TRATO VOCAL & ASPECTOS FONATÓRIOS & ASPECTOS DA DINÂMICA VOCAL \\
\hline \multirow[t]{2}{*}{8 (RASATI) } & & $\begin{array}{l}\text { Rouquidão: } 1 \\
\text { Aspereza: } 1 \\
\text { Soprosidade: } 2 \\
\text { Astenia: } 0 \\
\text { Tensão: } 1 \\
\text { Instabilidade: } 1 \\
\end{array}$ & \\
\hline & ASPECTOS DO TRATO VOCAL & ASPECTOS FONATÓRIOS & ASPECTOS DA DINÂMICA VOCAL \\
\hline 9 (VPAS) & $\begin{array}{l}\text { Ponta de língua avançada em grau 1, corpo de língua } \\
\text { elevado em grau } 1 \text { e com extensão diminuída em } \\
\text { grau } 2 \text {, mandíbula aberta em grau 1, hiperfunção } \\
\text { de trato vocal em grau 1, laringe alta em grau } 2 .\end{array}$ & $\begin{array}{l}\text { Voz crepitante em grau } \\
1 .\end{array}$ & $\begin{array}{l}\text { Pitch habitual elevado em grau } 2 \text { e loudness } \\
\text { habitual elevado em grau } 2 \text {. }\end{array}$ \\
\hline \multirow[t]{2}{*}{9 (RASATI) } & & $\begin{array}{l}\text { Rouquidão: } 0 \\
\text { Aspereza: } 0 \\
\text { Soprosidade: } 0 \\
\text { Astenia: } 0 \\
\text { Tensão: } 1 \\
\text { Instabilidade: } 0 \\
\end{array}$ & \\
\hline & ASPECTOS DO TRATO VOCAL & ASPECTOS FONATÓRIOS & ASPECTOS DA DINÂMICA VOCAL \\
\hline 10 (VPAS) & $\begin{array}{l}\text { Lábios com extensão diminuída em grau 1, } \\
\text { mandíbula com extensão diminuída em grau 1, } \\
\text { ponta de língua avançada em grau } 2 \text {. }\end{array}$ & \begin{tabular}{|l} 
Loudness habitual \\
diminuído em grau 1.
\end{tabular} & \\
\hline 10 (RASATI) & & $\begin{array}{l}\text { Rouquidão: } 0 \\
\text { Aspereza: } 0 \\
\text { Soprosidade: } 0 \\
\text { Astenia: } 0 \\
\text { Tensão: } 0 \\
\text { Instabilidade: } 0\end{array}$ & \\
\hline
\end{tabular}

Fonte: Vieira (2018)

\section{DISCUSSÃO}

A avaliação perceptivo-auditiva é considerada na clínica vocal como a mais efetiva e, mesmo que os profissionais se beneficiem de outras ferramentas e recursos, esta análise continua como uma das mais essenciais na avaliação e acompanhamento dos pacientes que apresentam distúrbios vocais ${ }^{12}$.

$\mathrm{Na}$ Fonoaudiologia, a avaliação vocal conta com diversos recursos avaliativos. Os mais utilizados baseiam-se na percepção auditiva dos avaliadores, sendo destacadas as escalas: RASATI ${ }^{4}$, VPAS-PB ${ }^{5}$, SVEA ${ }^{13}$ e o CAPE-V ${ }^{14}$.

A definição da qualidade de voz pode ser apresentada por duas vertentes, estreita e ampla: características de percepção de um lado e, do outro, características de produção. Sendo assim, a qualidade de voz daquela é vista como um aspecto único do processo de fonação, e como a resposta do ouvinte ao som da fala. Dessa forma, a qualidade vocal é o produto de uma complexa sequência de eventos acústicos, psicológicos e cognitivos, comumente chamado de "cadeia da fala"15 16.

Nos resultados da pesquisa Identificação de falante: um estudo perceptivo da qualidade de voz é possível observar a ampla descrição dos aspectos que compõem a fala. Além dos aspectos fonatórios, o VPAS-PB aborda aspectos de trato e dinâmica vocal, diferentemente do protocolo da RASATI, que se limita aos fonatórios.
Sendo a voz humana uma ferramenta importante para a comunicação, ela também constitui a personalidade do indivíduo, por meio da intensidade, projeção, qualidade vocal e modulação. Estas características interferem na eficácia da comunicação do falante ${ }^{17}$. Assim, a amostra avaliada pelo VPAS-PB possibilitou maior panorama sobre o perfil vocal do indivíduo, como identificado nos falantes audiogravados da pesquisa.

O profissional fonoaudiólogo atuante na área de voz deve ser capacitado para compreender as dimensões perceptivas, fisiológicas, psicológicas e acústicas durante o processo avaliativo. Dessa forma, seu julgamento perceptivo possibilitará um diagnóstico final mais adequado ${ }^{4}$.

Ao realizar a comparação entre os dois resultados, percebe-se que todos os falantes audiogravados, principalmente os de número 1, 3, 6, 8, 9 (Tabela 1) apresentaram uma descrição do perfil vocal mais detalhada por meio do VPAS-PB, do que a observada pela RASATI. Dessa forma, é possível observar a importância do uso do protocolo VPAS-PB no campo da perícia vocal em identificação de falantes, pela razão de detalhar outras características que a RASATI não detém.

O falante audiogravado de número 2, por exemplo, apresentou tremor e irregularidade laríngea e continuidade interrompida, que são aspectos de dinâmica vocal. Estes aspectos são apenas abordados no VPAS-PB, não sendo detectáveis em aplicação da RASATI, que se restringe a 
aspectos fonatórios. Por isso, em ambiente clínico, haveria uma falta de informação importante com o uso do protocolo da RASATI, assim como na identificação de indivíduos na perícia vocal, pois não forneceria todos os dados que compõem o perfil vocal do falante.

\section{CONCLUSÃO}

O VPAS-PB e a RASATI colaboram para a prática clínica do fonoaudiólogo, porém cabe a este profissional expandir a utilização de outros recursos durante a avaliação vocal. 0 VPAS-PB permite ao profissional maiores chances de identificar o problema vocal dentro dos aspectos apresentados, por abordar tanto trato vocal como fonte glótica, que é a base do protocolo da RASATI. Esta última, por sua vez, permite que $o$ avaliador identifique os aspectos fonatórios na prática clínica com maior velocidade, visto que aborda somente os aspectos que se referem à fonte glótica, mas não contempla os outros aspectos, como dinâmica vocal e trato vocal. Por isso, a utilização de um protocolo validado como a RASATI permite ao profissional uma avaliação de aspectos importantes da voz do indivíduo, mas que se restringem à fonte glótica. Dessa forma, ao fazer uso do VPAS-PB, outros aspectos seriam contemplados, evitando, assim, a perda de elementos importantes durante avaliação vocal.

\section{REFERÊNCIAS}

1. GREGIO, F. N. Configuração do trato vocal supraglótico na produção das vogais do português brasileiro: dados de imagens de ressonância magnética. 2006. Dissertação (Mestrado em Linguística Aplicada e Estudos da Linguagem) - Pontifícia Universidade Católica de São Paulo, São Paulo, 2006. Disponível em:

https://tede2.pucsp.br/bitstream/handle/13780/1/FABIANA\%20NOGUElRA\%20GREGIO.pdf. Acesso em: 03 out. 2019.

2. FANT, G. Acoustic theory of speech production. J. biomed. eng., Holanda, v. 4, n. 2, 2011. Disponível em: https://www.scirp. org/(S(czeh2tfqyw2orz553k1w0r45))/reference/ReferencesPapers. aspx?ReferencelD=89601. Acesso em: 07 out. 2019.

3. FUKUYAMA, E. E. Análise acústica da voz captada na faringe próximo à fonte glótica através de microfone acoplado ao fibrolaringoscópio. Rev. Bras. Otorrinolaringol., Rio de Janeiro, v. 67, n. 6, p.776-786, nov./dez. 2001. Disponível em: http://www.scielo.br/pdf/rboto/v67n6/8445.pdf. Acesso em: 03 mar. 2019.

4. PINHO, S. M. R.; PONTES, P. Escala de avaliação perceptiva da fonte glótica: RASAT. Vox Brasilis, São Paulo, v. 8, n. 3, p. 11-13, 2002. Disponível em: http://www.fonovim.com.br/arquivos/0a98d8cb14ab1362d29db01 10c7d4175-Disfonia-rasat-pt1.pdf. Acesso em: 03 mar. 2019.

5. SILVA, M. F. B. de L. et al. Avaliação de qualidade vocal: abordagem metodológica para análise de dados perceptivos. Rev. CEFAC, São Paulo, v. 19, n. 6, p.831-841, nov./dez. 2017. Disponível em: http://www.scielo. br/pdf/rcefac/v19n6/pt_1982-0216-rcefac-19-06-00831.pdf. Acesso em: 03 mar. 2019.
6. MARTENS, J.; VERSNEL, H.; DEJONCKERE, P. The effect of visible speech in the perceptual rating of pathological voices. J. Voice, v. 133, n. 2, p. 178-85, fev. 2007. Disponível em: https://www.ncbi.nlm.nih.gov/ pubmed/17309988. Acesso em: 07 out. 2019.

7. PINHO, S. M. R.; KORN, G. P.; PONTES, P. Desvendando os segredos da voz: músculos intrínsecos da laringe e dinâmica vocal. Rio de Janeiro: Revinter, 2008.

8. KREIMAN, J. et al. Individual differences in voice quality perception. J. speech lang. hear. res., Rockville, v. 35, n. 3, p. 512-520, June 1992. Disponivel em: https://www.researchgate.net/publication/21547975_Individual_Differences_in_Voice_Quality_Perception. Acesso em: 07 out. 2019.

9. PATEL, S.; SHRIVASTAV, R. Perception of dysphonic vocal quality: some thoughts and research update. Perspectives on Voice And Voice Disorders, [s.I], v. 17, n. 2, p.3-7, jul. 2007. Disponível em: https://www.researchgate. net/publication/235708494_Perception_of_Dysphonic_Vocal_Quality_Some_Thoughts_and_Research_Update. Acesso em: 07 out. 2019.

10. CAMARGO, Z.; MADUREIRA, S.; SCHMITZ, J. R. Qualidade vocal e produções de fala em três línguas: um estudo de caso. Rev. Intercâmbio, São Paulo, v. 27, p.110-140, 2013. Disponível em: https://revistas.pucsp. br/intercambio/article/view/17693/13190. Acesso em: 07 out. 2019.

11. VIEIRA, R. C. Identificação de falante: um estudo perceptivo da qualidade de voz. 2018. Tese (Doutorado em Línguística Aplicada e Estudos da Linguagem) - Pontifícia Universidade Católica de São Paulo, São Paulo, 2018. Disponível em: https://tede2.pucsp.br/bitstream/handle/20962/2/ Renata\%20Christina\%20Vieira.pdf. Acesso em: 03 out. 2019.

12. MUÑOZ, M. B. et al. Alterações fonoaudiológicas em crianças com hipotireoidismo congênito: revisão crítica da literatura. Rev. CEFAC, São Paulo, v. 16, n. 6, p. 2006-2014, nov./dez. 2014. Disponível em: http:// www.scielo.br/pdf/rcefac/v16n6/1982-0216-rcefac-16-06-02006.pdf. Acesso em: 03 out. 2019.

13. HAMmARBeRG, B. et al. Perceptual and acoustic correlates of abnormal voice qualities. Acta otolaryngol., Stockholm, v. 90, n. 5/6, p. 441-51, nov./dez.1980. Disponível em: https://www.ncbi.nlm.nih.gov/ pubmed/7211336. Acesso em: 07 out. 2019.

14. KEMPSTER, G. B. et al. Consensus auditoryperceptual evaluation of voice: Development of a standardized clinical protocol. Am j. speech lang. pathol., Rockville, v. 18, p.124-32, May 2009. Disponível em: https://pdfs.semanticscholar.org/feb9/283889b8152fd2c465887ad8f6c2dc1c0fe5.pdf?_ga =2.95204956.1534007799.15704768541044829515.1570476854. Acesso em: 07 out. 2019.

15. GOMES, M. L.; CARNEIRO, D.; DRESCH, A. Análise perceptiva e acústica em fonética forense: uma pesquisa em disfarce de voz. Domínios de Linguagem, Uberlândia, v. 10, n. 2, p.559-589, abr./jun. 2016. Disponível em: http://www.seer.ufu.br/index.php/dominiosdelinguagem/article/ view/31650/18491. Acesso em: 03 out. 2019.

16. KREIMAN, J.; SIDTIS, D. Foundations of voice studies: an interdisciplinary approach to voice. Estados Unidos: Wiley-Blackwell, 2011.

17. BARRETO, T. M. M. et al. Perfil da saúde vocal de cantores amadores de igreja evangélica. Rev. Soc. Bras. Fonoaudiol., Maceió, v. 16, n. 2, p.140145, 2011. Disponível em: http://www.scielo.br/pdf/rsbf/v16n2/06.pdf. Acesso em: 03 out. 2019.

Submetido em: 04/11/2019

Aceito em: 30/11/2019 
ANEXO 1

\begin{tabular}{|c|c|c|c|c|c|c|c|c|c|c|}
\hline \multirow[t]{3}{*}{ QUALIDADE VOCAL } & \multicolumn{2}{|c|}{ PRIMEIRA PASSADA } & \multicolumn{8}{|c|}{ SEGUNDA PASSADA } \\
\hline & \multirow[t]{2}{*}{ Neutro } & \multirow[t]{2}{*}{ Nāo neutro } & \multirow{2}{*}{\multicolumn{2}{|c|}{ AJUSTE }} & \multicolumn{3}{|c|}{ Moderado } & \multicolumn{3}{|c|}{ Extremo } \\
\hline & & & & & 1 & 2 & 3 & 4 & 5 & 6 \\
\hline \multicolumn{11}{|c|}{ A. ELEMENTOS DO TRATO VOCAL } \\
\hline \multirow{5}{*}{ 1.Lábios } & & & Arredondados/pr & otraidos & & & & & & \\
\hline & & & Estirados & & & & & & & \\
\hline & & & Labiodentalizaçá & & & & & & & \\
\hline & & & Extensáo diminu & & & & & & & \\
\hline & & & Extensấo aumen & tada & & & & & & \\
\hline & & & Fechada & & & & & & & \\
\hline 2. Mandibula & & & Aberta & & & & & & & \\
\hline & & & Protraida & & & & & & & \\
\hline & & & Extensấo diminu & & & & & & & \\
\hline & & & Extensấo aumen & tada & & & & & & \\
\hline 3.Lingua ponta/lamina & & & Avancada & & & & & & & \\
\hline & & & Recuada & & & & & & & \\
\hline 4. Corpo de língua & & & Avançado & & & & & & & \\
\hline & & & $\begin{array}{l}\text { Recuado } \\
\end{array}$ & & & & & & & \\
\hline & & & Elevado & & & & & & & \\
\hline & & & \begin{tabular}{|l|l} 
Abaixado \\
\end{tabular} & & & & & & & \\
\hline & & & Extensáo diminu & & & & & & & \\
\hline & & & Extensáo aumen & tada & & & & & & \\
\hline 5.Faringe & & & Constriçấo & & & & & & & \\
\hline & & & Expansáo & & & & & & & \\
\hline 6.Velofaringe & & & Escape nasal at & idivel & & & & & & \\
\hline & & & Nasal & & & & & & & \\
\hline & & & Denasal & & & & & & & \\
\hline 7. Altura de laringe & & & Elevada & & & & & & & \\
\hline & & & Abaixada & & & & & & & \\
\hline B. TENSAO MUSCULAR & & & & & & & & & & \\
\hline 8. Tensáo do trato vocal & & & Hiperfunçăo & & & & & & & \\
\hline & & & Hipofunçắo & & & & & & & \\
\hline 9. Tensáo laríngea & & & Hiperfunçăa & & & & & & & \\
\hline & & & Hipofunçắo & & & & & & & \\
\hline C. ELEMENTOS FONATO & & & & & & & & & & \\
\hline & AJUSTE & & Presente & & & us d & esce & & & \\
\hline & & & Neutro & Näo Neutro & & lera & & & emo & \\
\hline & & & & & 1 & 2 & 3 & 4 & 5 & 6 \\
\hline 10. Modo de fonaçáo & Modal & & & & & & & & & \\
\hline & Falsete & & & & & & & & & \\
\hline & Crepitáncia/ vocal & al fry & & & & & & & & \\
\hline & Voz crepitante & & & & & & & & & \\
\hline 11. Fricçăo laringea & Escape de ar & & & & & & & & & \\
\hline & Voz soprosa & & & & & & & & & \\
\hline 12.Irregularidade laringea & Voz áspera & & & & & & & & & \\
\hline DINÁMICA VOCAL & & Neutro & AJUSTE & & & der & & & remc & \\
\hline & & & & & 1 & 2 & 3 & 4 & 5 & 6 \\
\hline D. ELEMENTOS PR & OS & & & & & & & & & \\
\hline & Habitual & & Elevado & & & & & & & \\
\hline 13.Pitch (fo) & & & Abaixado & & & & & & & \\
\hline & Extensão & & Diminuída & & & & & & & \\
\hline & & & Aumentad & & & & & & & \\
\hline & Variabilidade & & Diminuida & & & & & & & \\
\hline & & & Aumentad & & & & & & & \\
\hline & Habitual & & Aumentad & & & & & & & \\
\hline 14.Loudness & & & Diminuído & & & & & & & \\
\hline (intensidade) & Extensão & & Diminuída & & & & & & & \\
\hline & & & Aumentad & & & & & & & \\
\hline & Variabilidade & & Diminuída & & & & & & & \\
\hline & & & Aumentad & & & & & & & \\
\hline 15. Tempo & & & & & & & & & & \\
\hline Continuidade & & & Interrompic & & & & & & & \\
\hline Taxa de elocução & & & Rápida & & & & & & & \\
\hline & & & Lenta & & & & & & & \\
\hline 16.OUTROS ELEM & & & & & & & & & & \\
\hline Suporte respiratório & & & Adequado & & & & & & & \\
\hline & & & Inadequad & & & & & & & \\
\hline
\end{tabular}




\section{ANEXO 2}

ESCALA RASATI

(Pinho e Pontes, 2008)

Nome:

Data de nascimento: Idade:

\section{GRADUAÇÃO}

\begin{tabular}{|c|c|c|c|c|c|c|}
\hline - Rouquidão: & ( ) 0 & ( ) 1 & ( ) $1-2$ & ( ) 2 & ( ) $2-3$ & ( ) 3 \\
\hline - Aspereza: & ( ) 0 & ( ) 1 & ( ) $1-2$ & ( ) 2 & ( ) $2-3$ & ( ) 3 \\
\hline - Soprosidade: & ( ) 0 & ( ) 1 & ( ) $1-2$ & ( ) 2 & ( ) 2-3 & ( ) 3 \\
\hline - Astenia: & ( ) 0 & ( ) 1 & ( ) $1-2$ & ( ) 2 & ( ) $2-3$ & ( ) 3 \\
\hline - Tensão: & ( ) 0 & ( ) 1 & ( ) $1-2$ & ( ) 2 & ( ) $2-3$ & ( ) 3 \\
\hline - Instabilidade: & $($ ) 0 & ( ) 1 & ( ) $1-2$ & $(\mathrm{l}) 2$ & ( ) 2-3 & $($ ) 3 \\
\hline
\end{tabular}

Graduação:

0 - ausente

1 - grau leve

1-2 - grau leve a moderado

2 - grau moderado

2-3 - grau moderado a intenso

3 - grau intenso

Data: 